\title{
Numerical wave propagation through ice-covered regions
}

\author{
Solvi Thrastarson ${ }^{1}$, Martin van Driel ${ }^{1}$, Michael Afanasiev ${ }^{1}$, and Andreas Fichtner ${ }^{1}$ \\ ${ }^{1}$ ETH Zürich. Institute of Geophysics, Sonneggstrasse 5. 8092, Zürich \\ Correspondence to: Solvi Thrastarson (soelvi.thrastarson@erdw.ethz.ch)
}

\section{A preprint submitted to eartharxiv.org}

\begin{abstract}
In recent years, seismic station coverage in polar regions has been increasing steadily, providing new insight into the deep structure and dynamics of these remote parts of the globe. Numerical seismic wave propagation through polar regions is complicated by the presence of ice sheets. At periods relevant for regional-scale waveform tomography, the ice acts as a thin layer that, for an exact solution, needs to be meshed by elements that are small compared to the minimum wavelength. As a
\end{abstract} consequence, computational costs may become prohibitively high.

In this study, we investigate the effects of various simplifications of the mesh using spectral-element simulations of regional seismic wave propagation, with propagation distances of several hundred kilometres. Our goals are to quantify errors in phase and amplitude, and to identify simplifications that significantly reduce computational costs while producing numerical errors that are tolerable given a concrete application, dataset and frequency band. Using the example of western Greenland, we specifically consider meshes where 1) the ice sheet is replaced by bedrock, 2) where the ice sheet is removed but bedrock topography is honored, and 3) where any topography and the ice sheet are ignored.

For periods of $10 \mathrm{~s}$ we find that the group velocity of fundamental-mode surface waves can be off by more than $2 \%$ when the ice sheet topography is ignored ( 2 and 3 ). The actual elastic properties of the ice play a comparatively smaller role. Furthermore, the ice sheet-related traveltime errors diminish with increasing period, whereas the topography-related errors are more frequency-independent. This suggests (1) that regional-scale topography with height much less than a wavelength should be modelled also in ice-free regions, and (2) that computational-costs may be reduced substantially by replacing ice with bedrock in the simulations, while fully honoring topography.

Amplitude errors in the fundamental-mode surface waves are equivalent to variations in attenuation that easily exceed $100 \%$, regardless of the type of simplification. It follows that the ice sheet should be meshed accurately when amplitude information is used to infer regional-scale Earth structure.

\section{Introduction}

Accurate structural models of the Earth are essential for the characterisation of earthquakes, the study of geodynamical processes, natural hazard assessment and exploration geophysics. Seismic tomography is used to image the Earth and to recover its seismically relevant parameters, such as velocities, attenuation, and density. Since the first applications of seismic tomography 
(e.g. Aki et al., 1977; Dziewonski et al., 1977), images of the Earth have improved greatly, thanks to the increasing volume and quality of data, but also as a result of methodological innovations. The latter include ray theory for arbitrary anisotropic media

(e.g. Cerveny, 1972; Červenỳ et al., 1977), finite-frequency kernels and tomography (e.g. Yomogida, 1992; Dahlen et al., 2000; Friederich, 2003; Yoshizawa and Kennett, 2004), ambient noise tomography (e.g. Shapiro and Campillo, 2004; Shapiro et al.,

5 2005; Sabra et al., 2005), and full-waveform inversion (FWI) (e.g. Igel et al., 1996; Chen et al., 2007; Fichtner et al., 2009; Tape et al., 2010).

To accurately image the Earth with FWI it is essential to produce synthetic data in the most accurate way possible with respect to the investigated wavelengths and computational resources available. In particular, properly accounting for the presence of topographically irregular ice is key to further progress in tomography for ice covered regions.

10 In this manuscript we aim to quantify the effect that ice sheets and topography can have on the regional wave field at periods down to $\sim 10 \mathrm{~s}$ and propagation distances on the order of $1000 \mathrm{~km}$. This is intended to assess the extent to which simplifications in the forward modelling, such as ignoring topography or replacing ice with bedrock, may be justified in the interest of lower computational requirements.

\subsection{Numerical modeling of wave propagation}

15 Accounting for the presence of ice and topography requires numerical methods for the solution of the seismic wave equation. Several of these methods are widely used, including the finite-difference (FD) method, employed since the late 1960's (Alterman and Karal, 1968; Virieux, 1984; Robertsson et al., 1994; Igel et al., 1995; Moczo et al., 2002). Various modifications of the method have been proposed to improve the handling of irregular topography and the free surface (e.g. Boore, 1970; Moczo, 1989; Robertsson, 1996; Kristek et al., 2002; Bohlen, 2002).

20 As an alternative to finite differences, the finite-element method (FEM) discretizes the medium by disjoint elements. In 3-D, the elements can either be tetrahedra, hexahedra or a mixture of both. The wave equation is approximated on each of the elements with the Galerkin method where the solution of the wave equation is represented by a finite superposition of basis functions. The elements can be aligned with boundaries and topography by using deformed shapes, and the sizes of the elements can be varied to account for the heterogeneous seismic medium. Based on the weak form of the equations of motion, FEM naturally accounts for the free surface and it can take any shape. Despite the flexibility of FEM, applications in seismology are comparatively rare (e.g. Lysmer and Drake, 1972; Bao et al., 1998). Reasons include the non-diagonality of the mass matrix which renders the implicit time stepping computationally expensive.

The spectral-element method (SEM) in the variant proposed by Faccioli et al. (1996, 1997), constitutes a modification of FEM where basis functions and collocation points for numerical integration are chosen to produce a diagonal mass matrix. Furthermore, SEM largely reduces Gibb's phenomenon, which leads to more rapid convergence with increasing polynomial degree, compared to regular FEM. Today, a wide range of SEM codes are available, all implementing a slightly different flavour, depending on specific target applications (e.g. Komatitsch and Tromp, 2002; Capdeville et al., 2003; Peter et al., 2011; Cupillard et al., 2012; Gokhberg and Fichtner, 2016; Nissen-Meyer et al., 2014).

For this study we use SEM for its accuracy and efficiency, and for its natural ability to handle complex media with topogra- 
phy and ice cover. Specifically, we employ the newly developed solver Salvus (Afanasiev et al., 2018). We prefer hexahedral elements because of substantially lower computational costs compared to the tetrahedral once (Cohen, 2003).

\subsection{Modelling wave propagation in ice-covered regions}

The challenge of modelling wave propagation in ice-covered regions is related to the presence of topography and the small thickness of the ice compared to the seismic wavelength.

At local (exploration) scales, Nuber et al. (2016) showed that neglecting topography with an amplitude fluctuation greater than half the minimum seismic wavelength leads to appreciable inversion artifacts. Similarly, Li et al. (2017) demonstrated the importance of including topography when inverting for S-wave velocity from Rayleigh wave dispersion curves. Although these studies were done for smaller scales and shorter wavelengths where the effect of topography is clearly larger than in our case, we will find that topography may need to be included for regional wave propagation at intermediate periods.

At periods of $\sim 10 \mathrm{~s}$, attainable in regional-scale waveform tomography (Tape et al., 2010; Fichtner and Villaseñor, 2015; Çubuk Sabuncu et al., 2017), the minimum wavelength is around $40 \mathrm{~km}$. This is one order of magnitude larger than the thickest ice sheets on Earth, reaching $\sim 4 \mathrm{~km}$ in central Antarctica (e.g. Drewry et al., 1982; Fretwell et al., 2013). As a consequence, the elements used to mesh the ice sheet are much smaller than those used to mesh the underlying crust and mantle. The small elements representing the ice sheet force a reduction of the minimum allowable time step in the numerical simulation, and therefore increase the computational costs significantly. In the following sections, we investigate simplifications of the structural model and the mesh in order to sidestep this limitation.

\subsection{Outline}

This manuscript is organized as follows: In section 2 we describe the meshing of an ice sheet using the example of Greenland, where the maximum ice thickness is around $3 \mathrm{~km}$. We propose several simplifications of the mesh that lead to computational savings, at the price of some numerical inaccuracies. Section 3 briefly describes measures of time and amplitude misfits that we then use in section 4 to quantify the time- and frequency-dependent numerical errors that result from the proposed simplifications. Finally, in section 5, we discuss the implications for regional-scale seismic tomography in ice covered regions, the potential reductions in computational requirements, as well as alternative approaches.

\section{Meshing and numerical wavefield simulations}

Without loosing generality, we focus this study on NW-Greenland. To build a numerical mesh, we begin with a spherical section, extending $400 \mathrm{~km}$ in depth, $1600 \mathrm{~km}$ in longitude, and $950 \mathrm{~km}$ in latitude, as shown in Figure 1. Material parameters for the mesh are taken from the isotropic version of the Preliminary Reference Earth Model (PREM, Dziewonski and Anderson, 1981). Subsequently, we deform the elements to honor topography, using the model of Hirt and Rexer (2015) with spherical harmonic degree up to 10800 . 


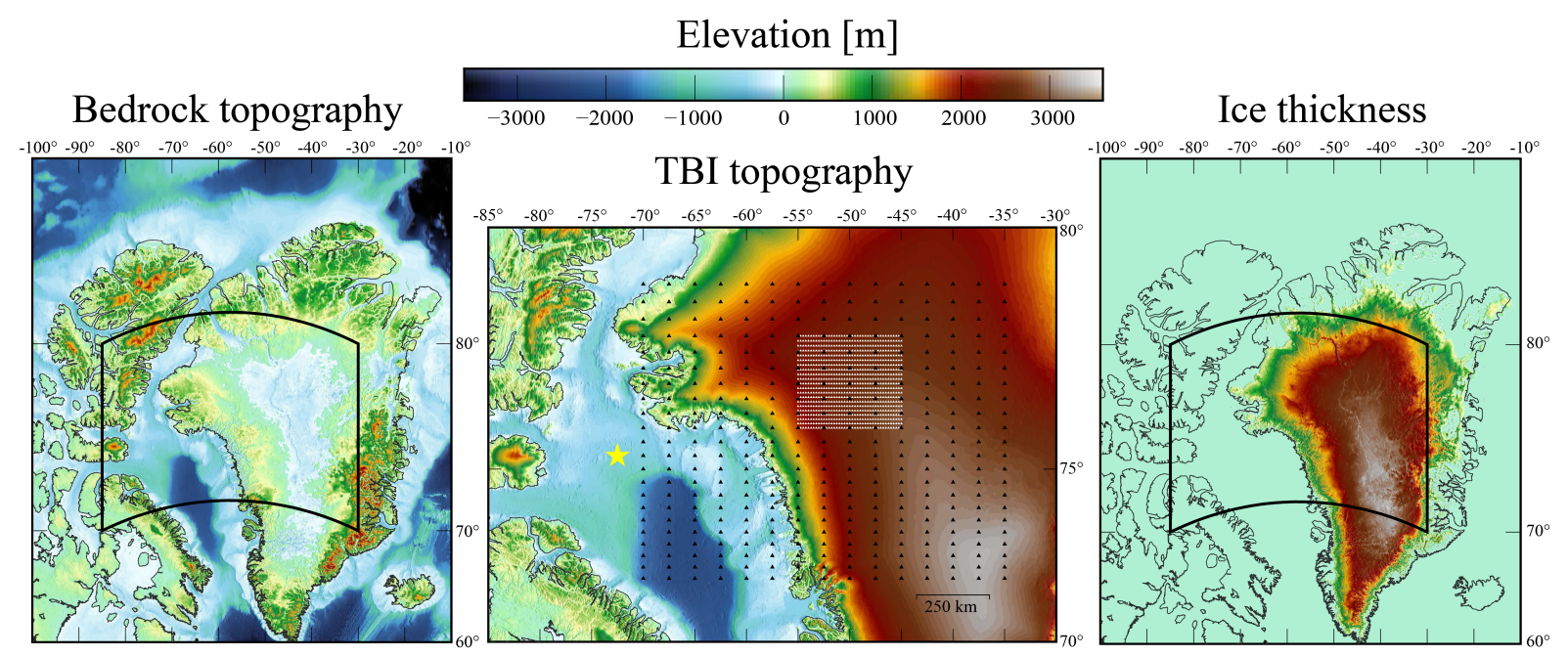

Figure 1. An overview of the studied area. The left panel displays the bedrock topography model from Hirt and Rexer (2015), the right panel shows the ice thickness model from the same source and the central panel has the two models summed together in what is called the Topography Bedrock Ice (TBI) model. The side panels show the surrounding area with the region used for simulations marked with black. The central panel illustrates the synthetic receiver configuration with black (coarse array) and white triangles (dense array) while the location of the simulated earthquake is marked with a yellow star.

\subsection{Meshing the ice sheet}

According to the work of Smith (1997), the P and S velocities of ice are around $v_{p}=3.84 \mathrm{~km} / \mathrm{s}$ and $v_{s}=1.95 \mathrm{~km} / \mathrm{s}$, respectively. Since the ice sheet has distinctly different elastic properties than the underlying bedrock, it must be implemented as an extra layer of elements. Owing to the CFL criterion (e.g. Courant et al., 1928; Quarteroni et al., 2000), the maximum allowed time step in the numerical simulations is controlled by the minimum grid point spacing within the entire mesh which is a function of the element size and the polynomial degree (4 in our case) used to approximate the wavefield (e.g. Fichtner, 2010; Igel, 2016).

We implemented the ice sheet by adding a layer of elements above what would normally be the top layer. The new layer is deformed in order to fit the Topography Bedrock Ice model of Hirt and Rexer (2015) (Figure 1). Elements with zero thickness in areas without ice sheet were subsequently deleted. A significant meshing problem occurs where the ice sheet becomes thin towards its edges. As the element size approaches zero, the available time step approaches zero as well. This limitation could potentially be overcome by meshing with tetrahedra. However, since hexahedra result in much faster simulations (e.g. Cohen, 2003), we opted for a pragmatic approach where elements thinner than $100 \mathrm{~m}$ are removed. The meshing process is illustrated in Figure 2. The ice sheet forces us to reduce the maximum allowable time step from $0.09 \mathrm{~s}$ to $0.002 \mathrm{~s}$, which corresponds to an increase of computational requirements by a factor of $\sim 45$. The explanation for this significant difference in allowable time step is that compared to the $100 \mathrm{~m}$ minimum element thickness in the ice sheet, the minimum thickness of an element which 
does not belong to the ice layer is $8.5 \mathrm{~km}$. This variation in minimum element thickness along with the velocity differences in the two layers $\left(v_{s}^{i c e}=1.95 \mathrm{~km} / \mathrm{s}\right.$ and $v_{p}^{r o c k}=3.5 \mathrm{~km} / \mathrm{s}$ ) explains the reduction in the allowable time step when the ice layer is included in the mesh.
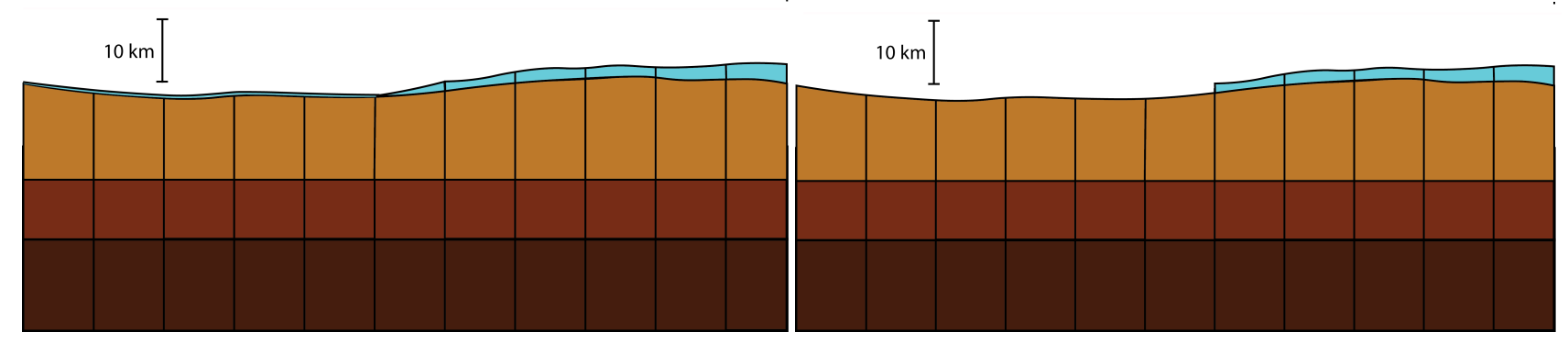

Figure 2. Schematic illustration of mesh building. The thin blue line in the left picture indicates a zero-thickness elements in areas without an ice sheet. Thin elements near the edge of the ice sheet were removed in order to obtain a computationally tractable time step.

\subsection{Mesh simplifications}

5 Although the edges of the ice sheet were removed, the limitations that the thickness of the ice sheet impose on the time step still remain. To overcome these limitations, we analyse the frequency-dependent numerical errors induced by three different simplifications. This will provide information on the application-dependent usefulness of the simplifications, and it will establish limits beyond which the full problem with the complex mesh needs to be solved.

The mesh where the ice sheet is fully implemented, described in section 2.1, serves as a reference mesh and will be compared to the simplified meshes. The first approximation made is to deform the bedrock to follow the topography of the ice, meaning that ice is replaced by bedrock of equal thickness. We refer to this simplification, illustrated in Figure 3, as S1. In S1, the extra layer of elements needed for the ice sheet is not necessary, thus bypassing the before-mentioned time step problem. The next level of simplification, referred to as S2, is to remove the ice sheet while still honoring bedrock topography (Figure 1). Finally, in the simplest possible mesh, we removed all topography and simply kept the 1D velocity model (S3).

By comparing the different simplifications to the reference, different ways in which the ice sheet influences the wavefield can be quantified. Comparison with S1 isolates the effect of the elastic parameters of the ice while the comparison with S2 recovers the combined effect of topography and the elastic parameters of the ice sheet. The comparison with S3 provides information on the general effect of topography/crustal thickness.

\subsection{Numerical wavefield simulation}

We simulate an earthquake that occurred on the 7th of July 2009 below Baffin Bay, off the west coast of Greenland (see Figure 1). The earthquake had a depth of $16.5 \mathrm{~km}$ and CMT magnitude 6.0 (Dziewonski et al., 1981; Ekström et al., 2012, www.globalcmt.org). To study the wavefield in detail as it crosses the boundary between ice-free and ice-covered regions, we 

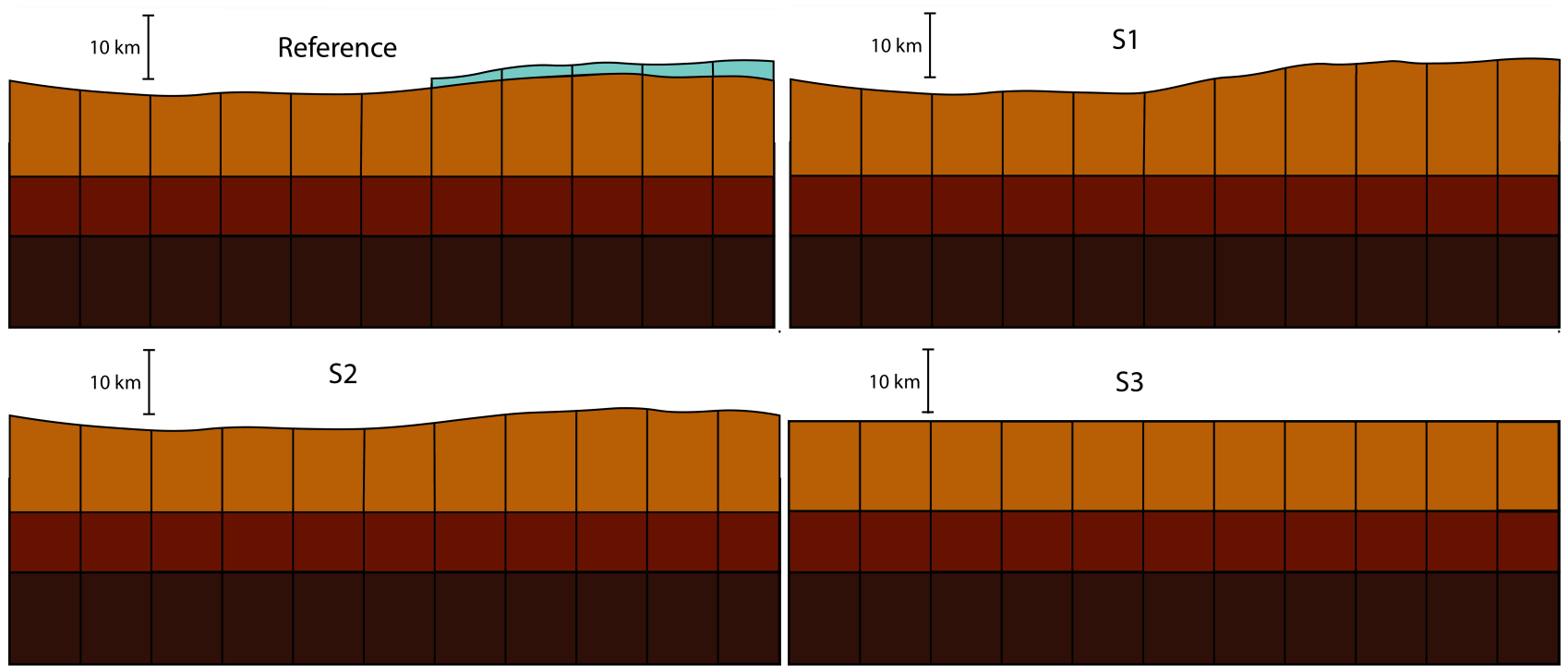

Figure 3. Schematic illustration of meshes. Top left is the reference mesh. Top right S1 (ice replaced by bedrock), bottom left S2 (ice removed) and bottom right S3 (any topography removed).

compute synthetic seismograms for 1523 receivers, shown in Figure 1).

As source time function, we used a Heaviside function, bandpass filtered between 10 and $120 \mathrm{~s}$. This corresponds to the period range that would typically be used in regional-scale waveform tomography (e.g. Tape et al., 2010; Zhu et al., 2012; Lee et al., 2014; Fichtner and Villaseñor, 2015; Simute et al., 2016). Since the duration of a magnitude 6 earthquake is usually below $5 \mathrm{~s}$

5 (e.g. Vallée and Douet, 2016), that is, below our minimum period, we ignored the source duration effect for this purely synthetic study. The wavefield simulation itself was performed using the SEM wave propagation solver Salvus (Afanasiev et al., 2018) on 96 CPUs of the high-performance computing cluster Piz Daint, operated by the Swiss National Supercomputing Center (CSCS).

\section{Waveform comparison}

10 To compare synthetic seismograms for the reference and the simplified meshes, we compute time- and frequency-dependent time shifts and amplitude differences, following the concept of Plonka et al. (2016). For this, we lowpass filter the time series to a specific minimum period $T_{\min }$. Subsequently, we apply a moving Gaussian window $\omega(t)$, with standard deviation equal to $T_{\min }$ to the filtered time series $u(t)$. This yields the windowed version $\hat{u}_{\tau}(t)=\omega(t-\tau) u(t)$, where $\tau$ is the moving center of the Gaussian window. To compute time shifts, we cross-correlate windowed synthetic seismograms from the simplified meshes with those from the reference mesh $\hat{u}_{\tau}^{\text {ref }}$. The time shift $\delta T$ is then defined as the argument of the maximum of the 
cross-correlation, that is,

$\delta T(\tau)=\operatorname{argmax}_{t} \int \hat{u}_{\tau}^{\mathrm{ref}}\left(t^{\prime}\right) \hat{u}_{\tau}\left(t+t^{\prime}\right) d t^{\prime}$.

The Gaussian window is then slid along the seismograms measuring $\delta T$ for every time window resulting in the time shift between the synthetic seismograms as a function of time. To stabilize the time shift measurements, we exclude windows where

5 the maximum amplitude is below $10 \%$ of the maximum amplitude of the complete time series.

To obtain a single-valued measure of extremal time shifts per trace, we will also use the signed maximum time $\operatorname{shift} \operatorname{absmax}(\delta T)=$ $\operatorname{sign}|\delta T| \max |\delta T|$. In section 4, we will see that the maximum time shift generally occurs within the fundamental-mode surface wave. The Gaussian window can also be used to measure amplitude misfits. For this, we compare the square root of the signal energy of the two seismograms within moving Gaussian windows, that is,

$\delta A(\tau)=\frac{\sqrt{\int\left[\hat{u}_{\tau}(t+\delta T(\tau))\right]^{2} d t}-\sqrt{\int\left[\hat{u}_{\tau}^{r e f}(t)\right]^{2} d t}}{\sqrt{\int\left[\hat{u}_{\tau}^{r e f}\right]^{2} d t}}$

To avoid biases of the amplitude misfit that result from time shifts, the time series are aligned prior to the computation of $\delta A(\tau)$.

We note that numerous alternative measures of seismic waveform differences have been proposed, often tuned to specific applications (e.g. Gee and Jordan, 1992; Kristeková et al., 2006; Fichtner et al., 2008; Kristekova et al., 2009; Bozdağ et al., 2011). Our decision to use the time and amplitude differences defined above is largely motivated by their simplicity, robustness and ease of interpretation.

\section{Results}

In the following we present an analysis of the ice sheet and topography effects. This is primarily done by using the subset of synthetic receivers shown in Figure 4, where time and amplitude misfits are computed at each of the stations. The stations have constant latitude and variable longitude, thereby allowing us to monitor misfit changes as the waves travel towards and across the ice sheet. For each of the simplified meshes we show time series and time-dependent misfits at receiver 5, right before the ice, and receiver 13, located $560 \mathrm{~km}$ behind the ice sheet boundary. Furthermore, the signed maximum time shift is displayed for each of the receivers along the line. To detect propagation off the great circle path or related to back scattering, we show the results of $f-k$ analyses performed with the dense array of synthetic receivers shown in Figure 1.

\subsection{No topography (S3)}

We first consider simplification S3, where all topography has been removed. Results are shown in Figure 5 for the full bandwidth of 10 to $120 \mathrm{~s}$. By completely neglecting topography, a negative time shift exceeding $1 \mathrm{~s}$ is introduced before the surface waves reach the ice. This means that the surface waves arrive earlier when topography is included. The topography west of the ice sheet is mostly negative meaning that the relatively slow velocity top layer is thinner when topography is included, thereby 


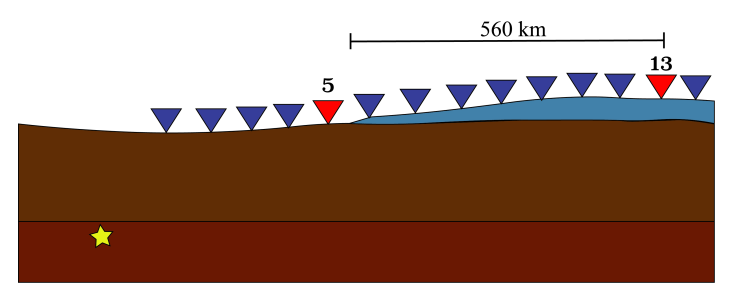

Figure 4. Schematic (not to scale) 2-D illustration of the stations used to quantify the effect of the ice sheet. Seismic stations are marked with triangles. Detailed time series and time-dependent misfits will only be displayed for the two receivers marked in red. The yellow star indicates the location of the earthquake.

leading to faster velocities, which explains the negative time shift.

This effect is reversed as the waves travel across the ice sheet. The topography becomes positive in the reference mesh and a lower-velocity ice layer is added on top of the bedrock. The extended bedrock layer and the ice layer introduce a steadily increasing misfit as the waves travel across. When the waves have traveled around $560 \mathrm{~km}$ across the ice sheet, a positive time shift approaching $5 \mathrm{~s}$ can be observed. If the initial negative time shift before reaching the ice sheet is taken into account, this corresponds to about $2.3 \%$ difference in surface wave velocity along the ray path across the ice sheet.

As detailed in section 4.4, time shifts generally increase with decreasing period, indicating a dispersion effect by the ice sheet. The higher frequency surface waves are more sensitive to the top layers, and adding a lower velocity layer on top of the bedrock increases the surface wave dispersion. Though time shifts are displayed only for the vertical component, the radial and tangential components display similar results, with maximum time shifts of $4-5 \mathrm{~s}$. The misfit for the tangential component is more localized in time as Love waves are mostly less dispersive than Rayleigh waves.

The time shift as a function of frequency of surface waves is quasi linear. It shows that if, in regional waveform tomography, an ice-sheet would be approximated as a flat surface with no topography, the inversion would yield artificial structural anomalies with intensities inversly proportional to depth.

15 The amplitudes of the recordings from S3 are generally up to $40 \%$ higher compared to the reference mesh. This could partly be due to inefficient transmission of energy into the ice layer, a longer propagation distance along an irregular surface, or scattering due to topography. The whole train of surface waves shows a similar difference in amplitude compared to the reference mesh showing that, when inverting for attenuation, neglecting the ice sheet would result in an artificial reduction in inferred attenuation.

\section{$20 \quad 4.2$ Bedrock topography (S2)}

For simplification S2, where the ice sheet is omitted but bedrock topography is honored, the negative time shift from the S3 comparison (before the surface waves reach the ice-covered region) has disappeared, as shown in Figure 6. This result is largely expected, as the $\mathrm{S} 2$ model is identical to the reference outside the ice-covered region. It indicates that missing back-scattering off the ice sheet plays a negligible role. 

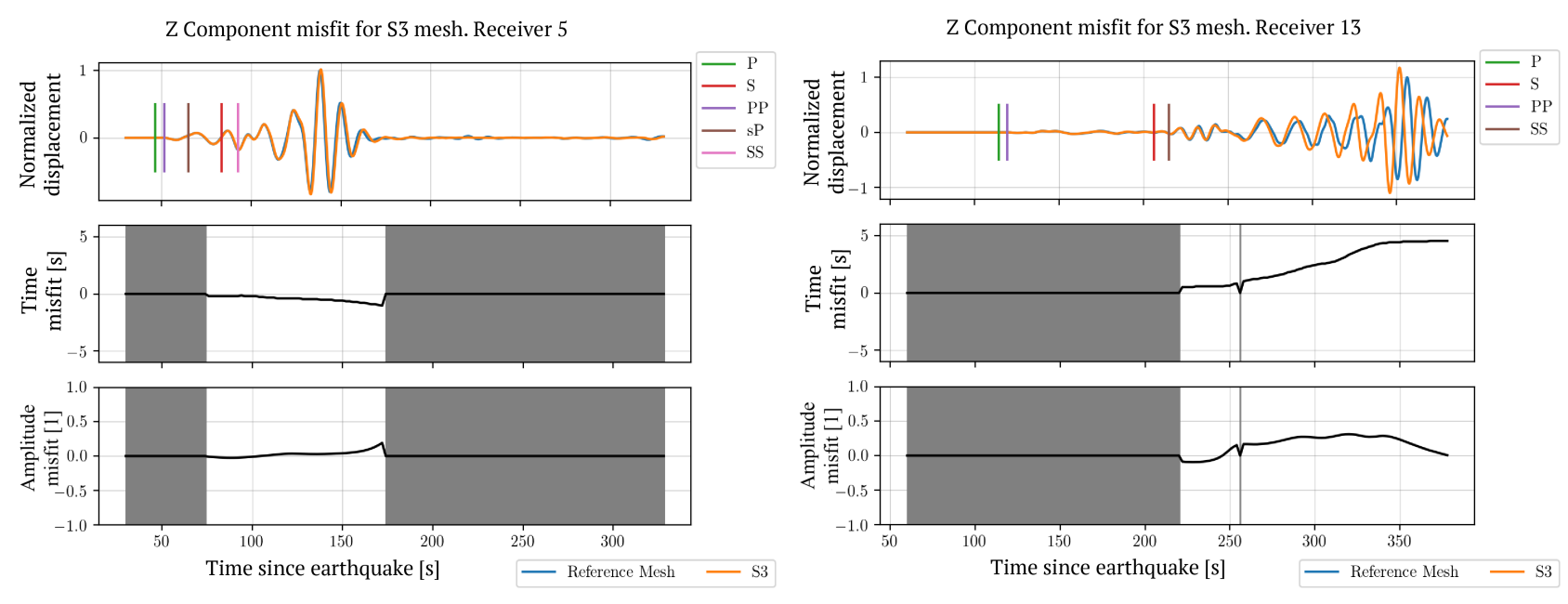

Figure 5. Time and amplitude misfits between vertical-component $(Z)$ synthetic seismograms computed for the reference mesh and the simplification S3 without any topography. Results are displayed for receivers 5 (left) and 13 (right). Colored vertical lines indicate the approximate arrival times of selected body wave phases, calculated using the taup package (Crotwell et al., 1999) implemented in ObsPy (Krischer et al., 2015; Beyreuther et al., 2010) and the 1D PREM model. Shaded areas indicate intervals where misfits were not computed because of too low amplitudes (below $10 \%$ of maximum amplitude). The waveforms are both normalized with respect to the maximum amplitude for the reference mesh.

Despite honoring bedrock topography, surface wave time shifts again accumulate to nearly $5 \mathrm{~s}$, as the wave propagates $560 \mathrm{~km}$ towards station 13. The S2 mesh causes a time shift that is slightly less than for the S3 mesh, but the improvement is minimal. Again, only the results for the vertical component are displayed, but the time shifts for the radial and tangential components are similar.

5 As for the S3 mesh, the amplitudes recorded for S2 are larger than for the reference, though the difference is slightly smaller. This supports the hypothesis that the difference is, at least partly, due to differences in scattering due to topography.

\subsection{Ice topography $(\mathrm{S} 1)$}

When the bedrock is deformed to fit the topography of the ice sheet (S1), the effect of the elastic parameters of the ice is effectively isolated. As shown in Figure 7, misfits are again negligible before the waves reach the ice sheet. At receiver 13, time shifts on the vertical component are mostly below $25 \%$ of the values for scenarios S2 and S3. The maximum time shift on the transverse component is slightly above $2 \mathrm{~s}$. This translates to effective velocity differences along the surface wave ray path of $0.6 \%$ (vertical) and $1.2 \%$ (transverse), respectively.

The time shift for the longer-period surface waves has faded while a time shift for the shorter period surface waves is still existent although it has diminished by $75 \%$. An inversion would thus not yield an artificial deep structure but rather a shallow structure. The inversion could thus yield a homogenised version of the ice sheet in the top layer of the model. 

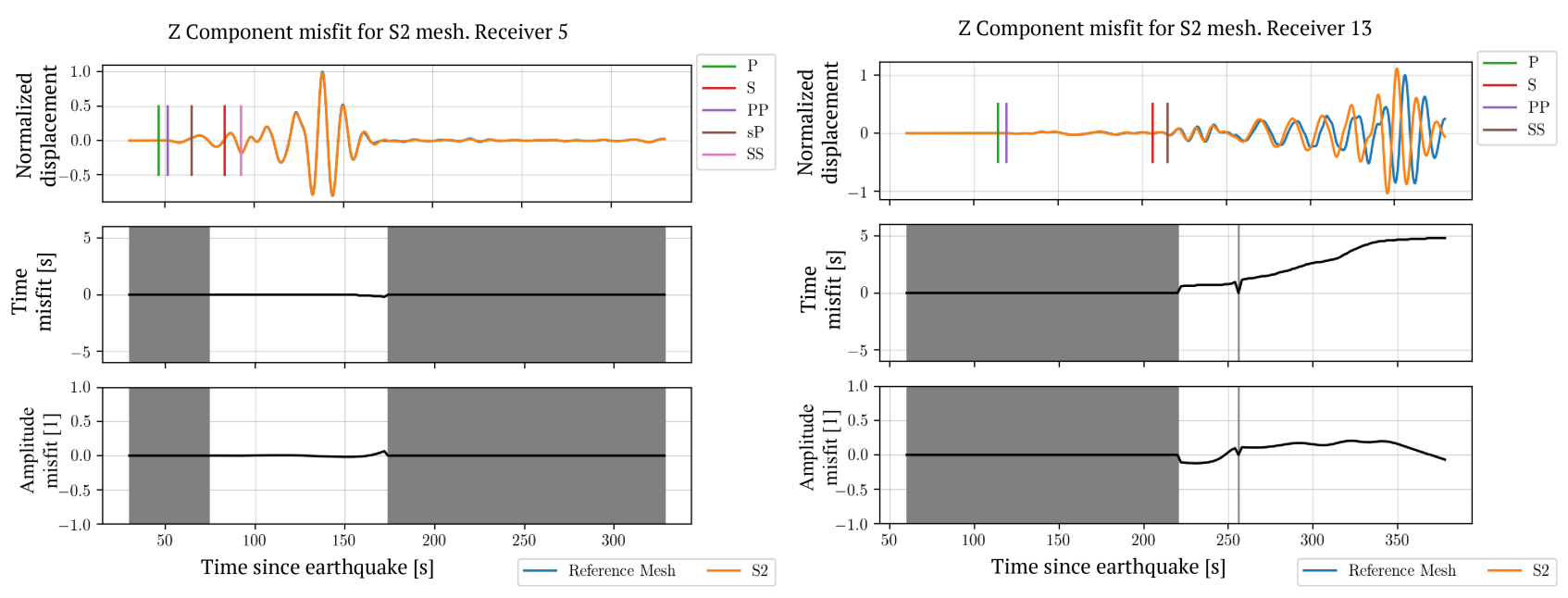

Figure 6. The same as Figure 5 but for simplification S2, where the ice sheet is omitted but bedrock topography is honored.

The amplitude misfit on the vertical component has diverged from the flat misfit resulting from the prior simplifications. This misfit pattern largely results from a change in waveform that is more significant than the previous examples. It suggests that S1 tends to affect dispersion (frequency dependence of phase velocity) in a more complex way than S2 and S3, which tend to perturb phase velocities in a more homogeneous manner as a function of frequency, thereby preserving the surface wave shape.

5 The same applies to the T component.

Table 1 summarizes maximum time shifts on the vertical, radial and transverse components for the three scenarios. It indicates that Rayleigh waves (vertical and radial) benefit most from the correct modelling of topography. Love waves (tangential) also improve, but their time shift remains clearly above the Rayleigh wave value due to the different elastic properties within the thin ice sheet. This observation is in accord with the depth sensitivity of surface wave phase velocity (e.g. Takeuchi et al., 1972), which has a maximum near the surface for Love waves, but a minimum for Rayleigh waves.

Table 1. Maximum signed time shifts for all components for all the simplified meshes.

\begin{tabular}{|l|r|r|r|}
\hline Mesh type & Vertical & Radial & Tangential \\
\hline S1 & $1.28 \mathrm{~s}$ & $1.34 \mathrm{~s}$ & $2.49 \mathrm{~s}$ \\
S2 & $4.80 \mathrm{~s}$ & $4.86 \mathrm{~s}$ & $4.48 \mathrm{~s}$ \\
S3 & $4.54 \mathrm{~s}$ & $4.54 \mathrm{~s}$ & $4.28 \mathrm{~s}$ \\
\hline
\end{tabular}

\subsection{Frequency dependence}

As a consequence of frequency-dependent sensitivity to Earth structure, misfits induced by various simplifications are frequencydependent as well. Figure 8 summarizes maximum signed time shifts for mesh $\mathrm{S} 1$ as a function of both the minimum period and the distance that surface waves travelled across the ice sheet. As expected, time shifts decrease with increasing period, 

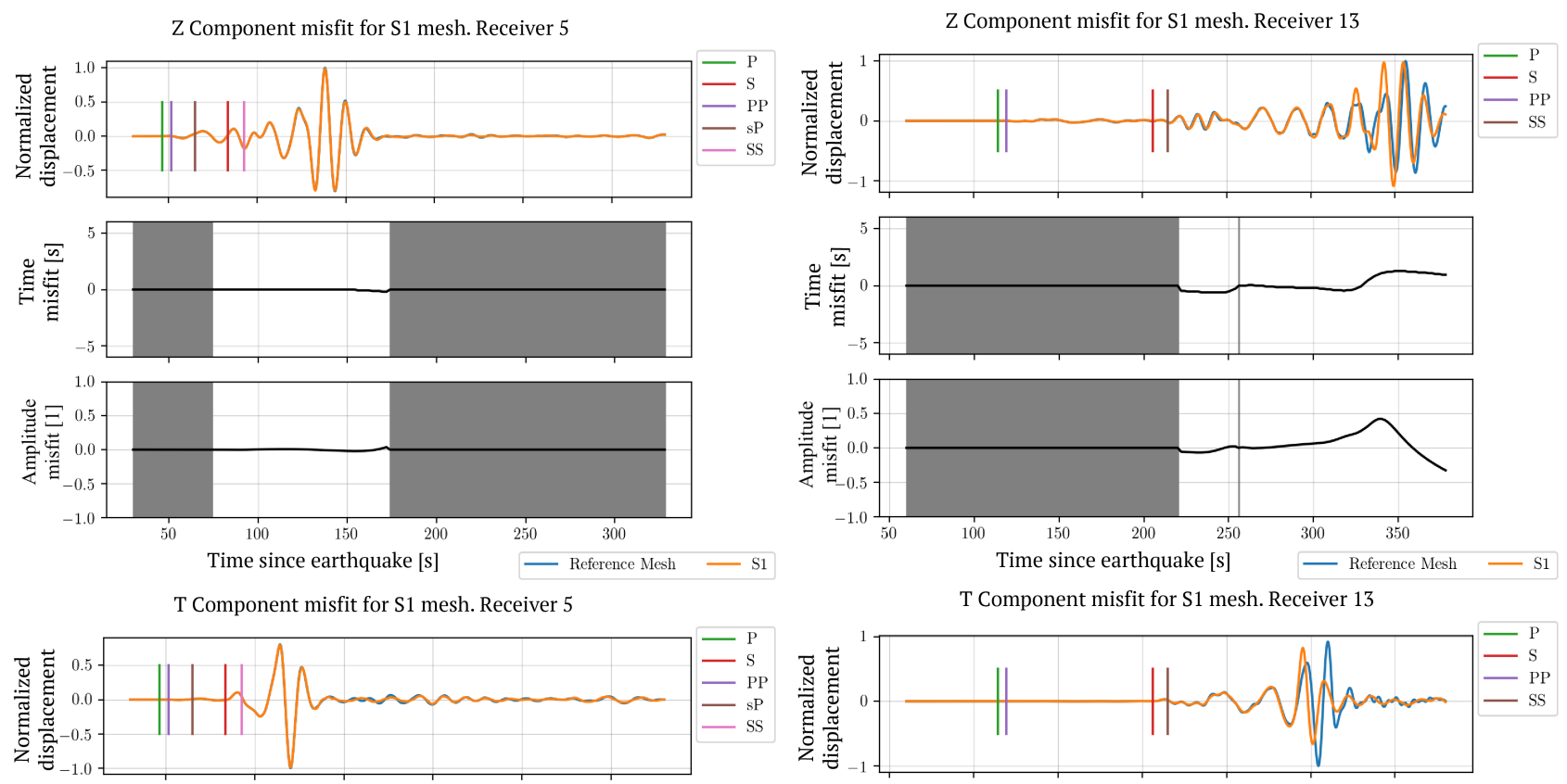

T Component misfit for S1 mesh. Receiver 13
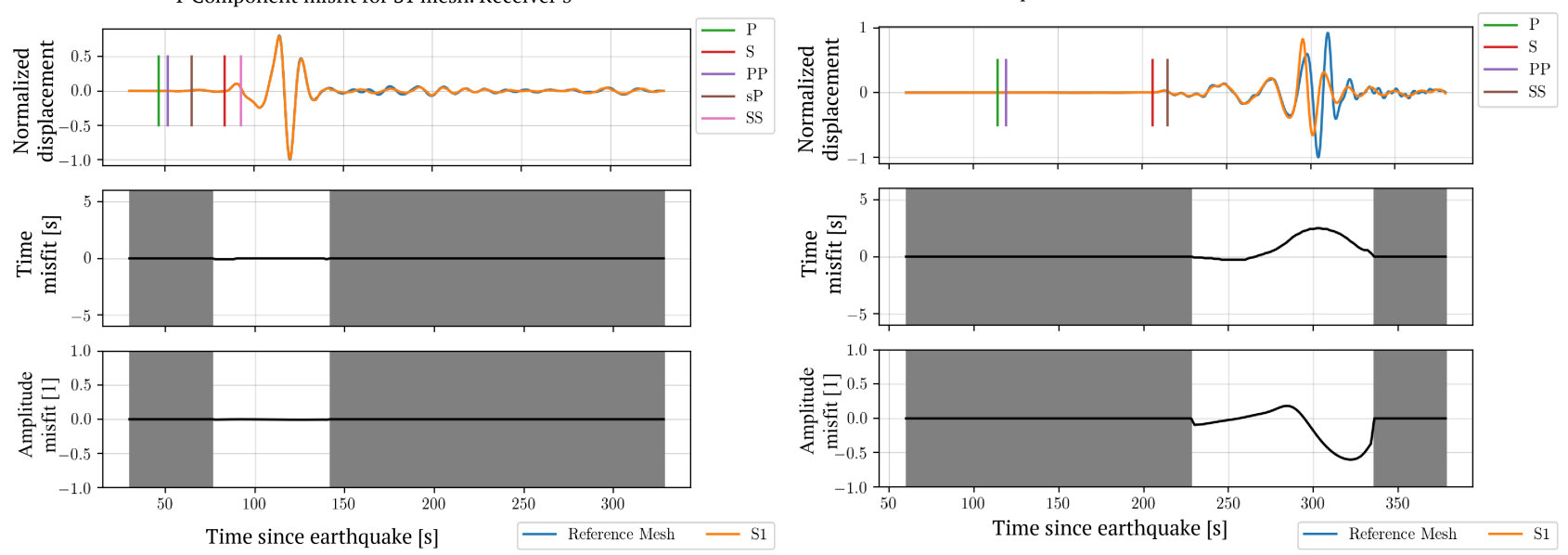

Figure 7. The same as Figure 5 but for simplification S1, where the ice sheet is replaced by bedrock, thus fully honoring topography. Vertical-component $(\mathrm{Z})$ seismograms are shown on top, transverse-component seismograms (T) below.

reaching $\sim 0.5 \mathrm{~s}$ after $560 \mathrm{~km}$ propagation for $25 \mathrm{~s}$ period. This beneficial effect may in practice be compensated by the increasingly featureless character of longer-period seismograms that carry less information on Earth structure. Misfit curves for the other scenarios are similar.

\subsection{FK analysis}

5 Using the seismic array illustrated with white triangles in Figure 1 an FK analysis (Capon and Bolt, 1973) was performed. FK analysis is an array processing technique where beamforming is performed for varying back azimuths and slownesses and the energy is calculated for each combination. The results can then be plotted on a circle with back azimuth on the azimuthal axis, slowness on the radial axis and relative energy as a colour scale. This was done to see whether any off great circle propagation 

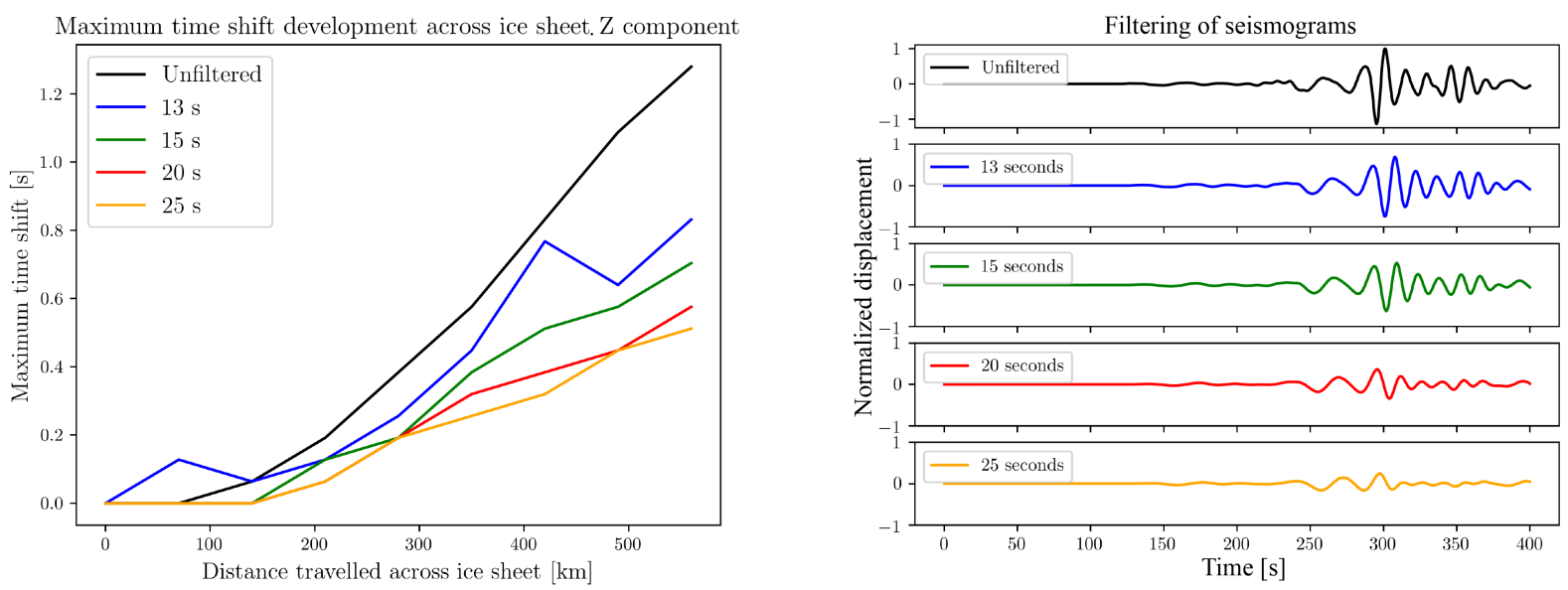

Figure 8. Maximum signed time shifts for scenario S1 as a function of minimum period and distance that surface waves travelled across the ice sheet. Filtered seismograms, shown to the right, become increasingly featureless for increasing periods.

paths could be detected related to scattering resulting from the ice sheet or guided waves travelling within the ice sheet. The results shown in Figure 9 show that no off great-circle propagation could be detected on any of the meshes and thus the ice sheet does not seem to influence the propagation direction of waves in this period range.
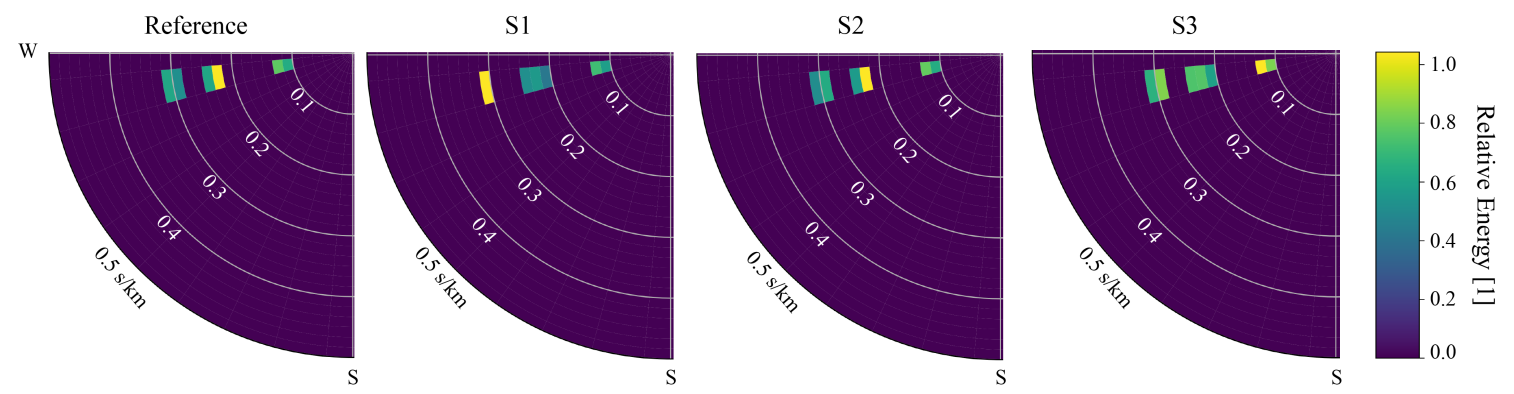

Figure 9. FK-Analysis of the Z-component recorded on the different meshes which shows the amount of energy measured by beamforming in terms of different slownesses and backazimuths. The plotted backazimuths are limited to $\mathrm{W}$ to $\mathrm{S}$ as the rest of the azimuth displayed zero energy.

\section{Discussion}

5 Depending on the simplifications made, traveltime misfits at $10 \mathrm{~s}$ period for surface waves correspond to fractional velocity variations, $\delta \ln v$, of $\sim 0.3$ to $2.3 \%$. Similarly, amplitude misfits correspond to absolute variations in attenuation, $\Delta Q^{-1}$, of $\sim 5 \cdot 10^{-3}$ to $20.0^{-3}$. (This corresponds to relative variations in attenuation). Both effects can significantly bias regional-scale 
tomography where velocity and attenuation heterogeneities are of that same order (e.g. Bezada et al., 2013; Bonnin et al., 2014; Palomeras et al., 2014; Schurr et al., 2003).

Avoiding these biases requires their detailed quantification, as well as a balanced choice involving application-dependent requirements on numerical accuracy, computational cost, and algorithmic complexity. These issues will be discussed in the following.

\subsection{Simplification- and frequency-dependent misfits}

Though body waves are affected by the mesh simplifications, the most significant effect is seen in surface waves, and Love waves in particular, due to their comparatively large sensitivity to shallow structure. For this reason, our discussion of time and amplitude misfits is mostly focused on surface waves.

10 A summary of the maximum signed time shifts at $10 \mathrm{~s}$ period as a function of distance travelled across the ice sheet is shown in the left panel of Figure 10 for the three scenarios considered. The comparison of meshes S2 and S3 with S1 at 10 s period suggests that the effect of topography is very substantial, roughly accounting for $4 \mathrm{~s}$ (out of $5 \mathrm{~s}$ ) in maximum time shift. The remaining $1 \mathrm{~s}$ for mesh S1 results from the elastic effect, that is, from replacing the properties of ice by the properties of bedrock.

15 As expected, maximum time shifts decrease with increasing minimum period, which is illustrated in the right panel of Figure 10. This improvement is most pronounced for scenario S1, but hardly relevant for S2 and S3. It follows that the simplification of topography cannot easily be compensated by increasing period.

While time shifts can be reduced significantly by accounting for topography, amplitude misfits are generally on the order of tens of percent for all three simplifications. This suggests that amplitude information should not be used, for instance, to constrain attenuation, unless the ice sheet is fully meshed.

\subsection{Computational Gain}

The main reason for using a simplified mesh are computational savings related to a potentially larger maximum time step in the numerical simulations. Table 2 displays the differences in computational cost, measured in CPU hours relative to the reference case, for the various mesh simplifications and for a minimum period of $10 \mathrm{~s}$. Replacing the ice sheet with bedrock (S3), reduces the computational cost by a factor of $1 / 0.0189 \approx 53$. This difference would increase for longer periods where the time step is still limited by the ice sheet in the reference mesh, whereas the elements in the simplified meshes could be enlarged. The absolute number of CPU hours is highly system-dependent. Running on 96 cores of Piz Daint, a Cray XC40/XC50 operated by the Swiss National Supercomputing Center (CSCS), required 155 CPU hours for the reference mesh and $400 \mathrm{~s}$ long synthetic seismograms. A typical full-waveform inversion requires at least 3 orders of magnitude more resources than a single forward simulation, meaning that the possible savings from using simplified meshes are very significant. We note that these numbers are of course specific to the scenarios considered here. They are intended to provide the rough order of magnitude. 

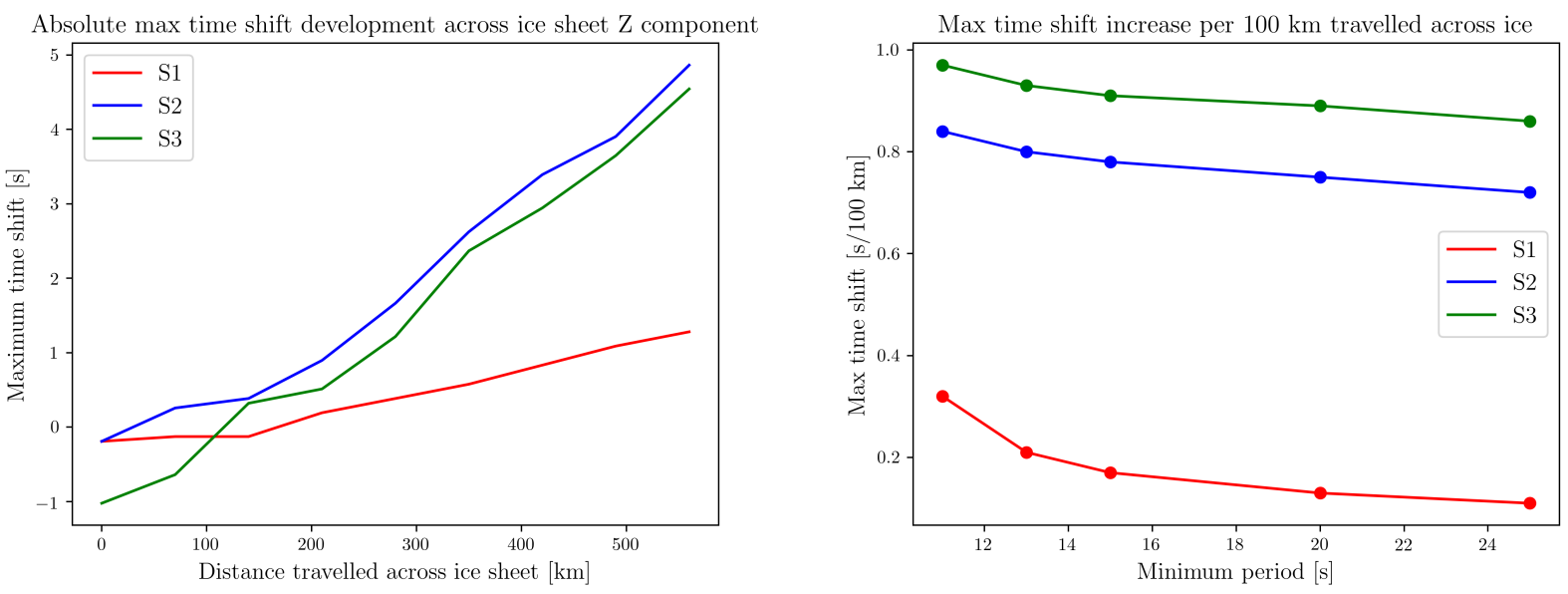

Figure 10. Maximum signed time shifts on the vertical component for the three different mesh simplifications. Left: Time shift at $10 \mathrm{~s}$ period as a function of distance travelled across the ice sheet. Right: Time shift increase per $100 \mathrm{~km}$ traveled across the ice sheet as a function of the minimum period.

Table 2. Number of elements, maximum time step and CPU hours (relative to the reference case) needed to compute $400 \mathrm{~s}$ long synthetic seismograms for the various mesh simplifications.

\begin{tabular}{|l|r|r|r|}
\hline Mesh type & Elements & Maximum time step [s] & CPU hours w.r.t. reference \\
\hline Reference & 171998 & 0.002 & 1.0 \\
S1 & 145920 & 0.09 & 0.0193 \\
S2 & 145920 & 0.09 & 0.0192 \\
S3 & 145920 & 0.09 & 0.0189 \\
\hline
\end{tabular}

\subsection{Alternative approaches}

In addition to the simplifications described in detail above, we also implemented a rock equivalent topography model (e.g. Hirt and Rexer, 2015) which replaces the ice sheet with bedrock of equal weight rather than equal thickness. This approach is similar to the modelling of an ocean layer by an equivalent weight (e.g. Komatitsch and Tromp, 2002), and may be justified theoretically as a first-order homogenization of a thin near-surface layer (Capdeville and Marigo, 2008). The results from these simulations produce time and amplitude misfits between those of scenarios S1 and S2, meaning that S1 would still be the preferred option.

Further improvements of the numerical solutions have been suggested in the context of homogenisation theory (Capdeville and Marigo, 2008, 2013). These include source and receiver corrections, as well as modifications of the free-surface boundary condition into a Dirichlet-to-Neumann condition. Especially in the presence of irregular topography, these approaches involve significant increases in algorithmic complexity. 
Finally, to reduce the computational bottleneck induced by small elements, various local time stepping schemes have been proposed (e.g. Dumbser et al., 2007; Rietmann et al., 2017). Their range of efficiency is, however, limited to meshes where the number of small elements is very small. This is not the case in our application where the ice sheet is meshed by several thousand elements.

\section{Conclusions}

We studied numerical seismic wave propagation through ice covered regions at scales of several hundred kilometers and periods of $10 \mathrm{~s}$ and more. This was intended to identify simplifications in the numerical mesh that can reduce computational costs and thereby contribute to the feasibility of seismic waveform tomography in polar regions.

From our simulations we can draw the following conclusions: (1) As expected, fundamental-mode surface waves, and Love waves in particular, are most affected by mesh simplifications where the ice sheet is removed. (2) Most of the traveltime errors of Rayleigh waves, around $75 \%$, are due to simplifications in topography. This suggests that traveltime errors may be reduced to a tolerable level by replacing ice with bedrock, while fully honoring topography. Of course, this will depend on the properties of a concrete dataset. (3) The actual elastic properties of the ice play a comparatively smaller role. Also, in contrast to the topography-related effect, the elastic effect of the thin sheet decreases with increasing period. (4) Amplitude errors in

15 the fundamental-mode surface waves are generally large, exceeding tens of percent, unless the ice sheet itself is meshed. In the context of regional seismic waveform tomography we conclude that topography of the ice should be honored to obtain reliable surface wave traveltimes. This statement holds also for ice-free regions and even when topographic variations are only a fraction of the minimum wavelength (around $10 \%$ in our case). Furthermore, the ice sheet itself should be meshed in case amplitude information is used to infer Earth structure.

Acknowledgements. This work was supported by the CSCS computing time grants ch1, s741 and s830, the European Research Council (ERC) under the EU's Horizon 2020 programme (grant No. 714069), and by the PASC Project GeoScale. 


\section{References}

Afanasiev, M., Boehm, C., van Driel, M., Krischer, L., amd D. A. May, M. R., Knepley, M. G., and Fichtner, A.: Modular and flexible spectral-element waveform modeling in two and three dimensions, Geophys. J. Int., submitted, 2018.

Aki, K., Christoffersson, A., and Husebye, E. S.: Determination of the three-dimensional seismic structure of the lithosphere, Journal of Geophysical Research, 82, 277-296, 1977.

Alterman, Z. and Karal, F.: Propagation of elastic waves in layered media by finite difference methods, Bulletin of the Seismological Society of America, 58, 367-398, 1968.

Bao, H., Bielak, J., Ghattas, O., Kallivokas, L. F., O’Hallaron, D. R., Shewchuk, J. R., and Xu, J.: Large-scale simulation of elastic wave propagation in heterogeneous media on parallel computers, Computer Methods in Applied Mechanics and Enigneering, 152, 85-102, 1998.

Beyreuther, M., Barsch, R., Krischer, L., Megies, T., Behr, Y., and Wassermann, J.: ObsPy: A Python toolbox for seismology, Seismological Research Letters, 81, 530-533, 2010.

Bezada, M., Humphreys, E., Toomey, D., Harnafi, M., Dávila, J., and Gallart, J.: Evidence for slab rollback in westernmost Mediterranean from improved upper mantle imaging, Earth Planet. Sci. Lett, 368, 51-60, 2013.

15 Bohlen, T.: Parallel 3-D viscoelastic finite difference seismic modelling, Computers \& Geosciences, 28, 887-899, 2002.

Bonnin, M., Nolet, G., Villasenor, A., Gallart, J., and Thomas, C.: Multiple-frequency tomography of the upper mantle beneath the African/Iberian collision zone, Geophysical Journal International, 198, 1458-1473, 2014.

Boore, D. M.: Love waves in nonuniform wave guides: finite difference calculations, Journal of Geophysical Research, 75, 1512-1527, 1970.

Bozdağ, E., Trampert, J., and Tromp, J.: Misfit functions for full waveform inversion based on instantaneous phase and envelope measurements, Geophys. J. Int., 185, 845-870, 2011.

Capdeville, Y. and Marigo, J.-J.: Shallow layer correction for spectral element like methods, Geophysical Journal International, 172, 11351150,2008

Capdeville, Y. and Marigo, J.-J.: A non-periodic two-scale asymptotic method to take account of rough topographies for 2-D elastic wave propagation, Geophys. J. Int., 192, 163-189, 2013.

Capdeville, Y., Chaljub, E., Vilotte, J. P., and Montagner, J. P.: Coupling the Spectral Element Method with a modal solution for Elastic Wave Propgation in Global Earth Models, Geophysical Journal International, 152, 34-66, 2003.

Capon, J. and Bolt, B.: Signal processing and frequency-wavenumber spectrum analysis for a large aperture seismic array, Methods in Computational Physics (Elsevier, 1973), 13, 1-59, 1973.

Çubuk Sabuncu, Y., Taymaz, T., and Fichtner, A.: 3-D crustal velocity structure of western Turkey: Constraints from full-waveform tomography, Phys. Earth Planet. Int., 270, 90-112, 2017.

Cerveny, V.: Seismic rays and ray intensities in inhomogeneous anisotropic media, Geophysical Journal International, $29,1-13,1972$.

Červenỳ, V., Molotkov, I., Molotkov, I., and Pšenč́́k, I.: Ray method in seismology, Univerzita Karlova, 1977.

Chen, P., Zhao, L., and Jordan, T. H.: Full 3D tomography for the crustal structure of the Los Angeles region., Bulletin of the Seismological Society of America, 97, 1094-1120, 2007.

35 Cohen, G. C.: Higher-order numerical methods for transient wave equations, 2003.

Courant, R., Friedrichs, K., and Lewy, H.: Über die partiellen Differenzengleichungen der mathematischen Physik, Mathematische annalen, 100, 32-74, 1928. 
Crotwell, H. P., Owens, T. J., and Ritsema, J.: The TauP Toolkit: Flexible seismic travel-time and ray-path utilities, Seismological Research Letters, 70, 154-160, 1999.

Cupillard, P., Delavaud, E., Burgos, G., Festa, G., Vilotte, J.-P., Capdeville, Y., and Montagner, J.-P.: RegSEM: a versatile code based on the spectral element method to compute seismic wave propagation at the regional scale, Geophysical Journal International, 188, 1203-1220, 2012.

Dahlen, F., Hung, S.-H., and Nolet, G.: Fréchet kernels for finite-frequency traveltimes-I. Theory, Geophysical Journal International, 141, 157-174, 2000.

Drewry, D. J., Jordan, S. R., and Jankowski, E.: Measured properties of the Antarctic ice sheet: surface configuration, ice thickness, volume and bedrock characteristics, Annals of Glaciology, 3, 83-91, 1982.

Dumbser, M., Käser, M., and Toro, E.: An arbitrary high-order discontinuous Galerkin method for elastic waves on unstructures meshes, Part V: Local time stepping and $p$-adaptivity, Geophys. J. Int., 171, 695-717, 2007.

Dziewonski, A., Chou, T.-A., and Woodhouse, J.: Determination of earthquake source parameters from waveform data for studies of global and regional seismicity, Journal of Geophysical Research: Solid Earth, 86, 2825-2852, 1981.

Dziewonski, A. M. and Anderson, D. L.: Preliminary reference Earth model, Physics of the earth and planetary interiors, 25, 297-356, 1981.

Dziewonski, A. M., Hager, B. H., and O'Connell, R. J.: Large-scale heterogeneities in the lower mantle, Journal of Geophysical Research, $82,239-255,1977$.

Ekström, G., Nettles, M., and Dziewoński, A.: The global CMT project 2004-2010: Centroid-moment tensors for 13,017 earthquakes, Physics of the Earth and Planetary Interiors, 200, 1-9, 2012.

Faccioli, E., Maggio, F., Quarteroni, A., and Tagliani, A.: Spectral-domain decomposition methods for the solution of acoustic and elastic wave equations, Geophysics, 61:4, 1160-1174, 1996.

Faccioli, E., Maggio, F., Paolucci, R., and Quarteroni, A.: 2D and 3D elastic wave propagation by a pseudo-spectral domain decomposition method, Journal of seismology, 1, 237-251, 1997.

Fichtner, A.: Full Seismic Waveform Modelling and Inversion., Springer, Heidelberg., 2010.

Fichtner, A. and Villaseñor, A.: Crust and upper mantle of the western Mediterranean - Constraints from full-waveform inversion, Earth Planet. Sci. Lett., 428, 52-62, 2015.

Fichtner, A., Kennett, B. L. N., Igel, H., and Bunge, H.-P.: Theoretical background for continental- and global-scale full-waveform inversion in the time-frequency domain., 175, 665-685, 2008.

Fichtner, A., Kennett, B. L. N., Igel, H., and Bunge, H.-P.: Full seismic waveform tomography for upper-mantle structure in the Australasian region using adjoint methods., Geophysical Journal International, 179, 1703-1725, 2009.

30 Fretwell, P., Pritchard, H. D., Vaughan, D. G., Bamber, J. L., Barrand, N. E., Bell, R., Bianchi, C., Bingham, R. G., Blankenship, D. D., Casassa, G., Catania, G., Callens, D., Conway, H., Cook, A. J., Corr, H. F. J., Damaske, D., Damm, V., Ferraccioli, F., Forsberg, R., Fujita, S., Gim, Y., Gogineni, P., Griggs, J. A., Hindmarsh, R. C. A., Holmlund, P., Holt, J. W., Jacobel, R. W., Jenkins, A., Jokat, W., Jordan, T., King, E. C., Kohler, J., Krabill, W., Riger-Kusk, M., Langley, K. A., Leitchenkov, G., Leuschen, C., Luyendyk, B. P., Matsuoka, K., Mouginot, J., Nitsche, F. O., Nogi, Y., Nost, O. A., Popov, S. V., Rignot, E., Rippin, D. M., Rivera, A., Roberts, J., Ross, N., Siegert, M. J.,

Smith, A. M., Steinhage, D., Studinger, M., Sun, B., Tinto, B. K., Welch, B. C., Wilson, D., Young, D. A., Xiangbin, C., and Zirizzotti, A.: Bedmap2: improved ice bed, surface and thickness datasets for Antarctica, The Cryosphere, 7, 375-393, https://doi.org/10.5194/tc-7375-2013, https://www.the-cryosphere.net/7/375/2013/, 2013. 
Friederich, W.: The S-velocity structure of the East Asian mantle from inversion of shear and surface waveforms, Geophysical Journal International, 153, 88-102, 2003.

Gee, L. S. and Jordan, T. H.: Generalized seismological data functionals, Geophys. J. Int., 111, 363-390, 1992.

Gokhberg, A. and Fichtner, A.: Full-waveform inversion on heterogeneous HPC systems, Computers and Geosciences, 89, 260-268, 2016.

5 Hirt, C. and Rexer, M.: Earth2014: 1 arc-min shape, topography, bedrock and ice-sheet models-available as gridded data and degree-10,800 spherical harmonics, International Journal of Applied Earth Observation and Geoinformation, 39, 103-112, 2015.

Igel, H.: Computational seismology: A practical introduction, Oxford University Press, 2016.

Igel, H., Mora, P., and Riollet, B.: Anisotropic wave propagation through finite-difference grids, Geophysics, 60, $1203-1216,1995$.

Igel, H., Djikpesse, H., and Tarantola, A.: Waveform inversion of marine reflection seismograms for P impedance and Poisson's ratio., Geophysical Journal International, 124, 363-371, 1996.

Komatitsch, D. and Tromp, J.: Spectral-element simulations of global seismic wave propagation, Part II: 3-D models, oceans, rotation, and gravity, Geophysical Journal International, 150, 303-318, 2002.

Krischer, L., Megies, T., Barsch, R., Beyreuther, M., Lecocq, T., Caudron, C., and Wassermann, J.: ObsPy: A bridge for seismology into the scientific Python ecosystem, Computational Science \& Discovery, 8, 014 003, 2015.

Kristek, J., Moczo, P., and Archuleta, R. J.: Efficient methods to simulate planar free surface in the 3D 4th-order staggered-grid finitedifference schemes, Studia Geophysica et Geodaetica, 46, 355-381, 2002.

Kristeková, M., Kristek, J., Moczo, P., and Day, S. M.: Misfit criteria for quantitative comparison of seismograms, Bulletin of the seismological Society of America, 96, 1836-1850, 2006.

Kristekova, M., Kristek, J., and Moczo, P.: Time-frequency misfit and goodness-of-fit criteria for quantitative comparison of time signals, Geophys. J. Int., 178, 813-825, 2009.

Lee, E.-J., Chen, P., Jordan, T. H., Maechling, P. B., Denolle, M., and Beroza, G. C.: Full-3D tomography (F3DT) for crustal structure in Southern California based on the scattering-integral (SI) and the adjoint-wavefield (AW) methods, J. Geophys. Res., 119, doi: 10.1002/2014JB011346, 2014.

Li, J., Schuster, G. T., Lin, F.-C., and Alam, A.: Wave-equation dispersion inversion of surface waves recorded on irregular topography, in: SEG Technical Program Expanded Abstracts 2017, pp. 2621-2626, Society of Exploration Geophysicists, 2017.

Lysmer, J. and Drake, L. A.: A finite-element method for seismology, Methods in Computational Physics, 11, 181-216, 1972.

Moczo, P.: Finite-difference technique for SH-waves in 2-D media using irregular grids-application to the seismic response problem, Geophysical Journal International, 99, 321-329, 1989.

Moczo, P., Kristek, J., Vavrycuk, V., Archuleta, R., and Halada, L.: 3D heterogeneous staggered-grid finite-difference modeling of seismic motion with volume harmonic and arithmetic averaging of elastic moduli, Bulletin of the Seismological Society of America, 92, 30423066, 2002.

Nissen-Meyer, T., Driel, M. v., Stähler, S., Hosseini, K., Hempel, S., Auer, L., Colombi, A., and Fournier, A.: AxiSEM: broadband 3-D seismic wavefields in axisymmetric media, Solid Earth, pp. 425-445, 2014.

Nuber, A., Manukyan, E., and Maurer, H.: Ground topography effects on near-surface elastic full waveform inversion, Geophysical Journal International, 207, 67-71, 2016.

Palomeras, I., Thurner, S., Levander, A., Liu, K., Villaseñor, A., Carbonell, R., and Harnafi, M.: Finite-frequency Rayleigh wave tomography of the western Mediterranean: Mapping its lithospheric structure, Geochemistry, Geophysics, Geosystems, 15, 140-160, 2014. 
Peter, D., Komatitsch, D., Luo, Y., Martin, R., Le Goff, N., Casarotti, E., Le Loher, P., Magnoni, F., Liu, Q., Blitz, C., Nissen-Meyer, T., Basini, P., and Tromp, J.: Forward and adjoint simulations of seismic wave propagation on fully unstructured hexahedral meshes, Geophysical Journal International, 186, 721-739, 2011.

Plonka, A., Blom, N., and Fichtner, A.: The imprint of crustal density heterogeneities on regional seismic wave propagation, Solid Earth, 7, 1591, 2016.

Quarteroni, A., Sacco, R., and Saleri, F.: Numerical Mathematics, Springer, New York, 2000.

Rietmann, M., Grote, M., Peter, D., and Schenk, O.: Newmark local time stepping on high-performance computing architectures, J. Comp. Phys., 334, 308-326, 2017.

Robertsson, J., Blanch, J., and Symes, W.: Viscoelastic finite-difference modeling, Geophysics, 59, 1444-1456, 1994.

Robertsson, J. O.: A numerical free-surface condition for elastic/viscoelastic finite-difference modeling in the presence of topography, Geophysics, 61, 1921-1934, 1996.

Sabra, K. G., Gerstoft, P., Roux, P., and Kuperman, W. A.: Surface wave tomography from microseisms in Southern California, Geophysical Research Letters, 32, doi:10.1029/2005GL023 155, 2005.

Schurr, B., Asch, G., Rietbrock, A., Trumbull, R., and Haberland, C.: Complex patterns of fluid and melt transport in the central Andean subduction zone revealed by attenuation tomography, Earth and Planetary Science Letters, 215, 105-119, 2003.

Shapiro, N. M. and Campillo, M.: Emergence of broadband Rayleigh waves from correlations of the ambient seismic noise, Geophysical Research Letters, 31, 2004.

Shapiro, N. M., Campillo, M., Stehly, L., and Ritzwoller, M.: High resolution surface wave tomography from ambient seismic noise, Science, 307, 1615-1618, 2005.

20 Simute, S., Steptoe, H., Gokhberg, A., and Fichtner, A.: Full-waveform inversion of the Japanese islands region, J. Geophys. Res., 121, 3722-3741, 2016.

Smith, A.: Basal conditions on Rutford ice stream, West Antarctica, from seismic observations, Journal of Geophysical Research: Solid Earth, 102, 543-552, 1997.

Takeuchi, H., Saito, M., and Bolt, B.: Seismic surface waves, Methods in computational physics, 11, 217-295, 1972.

Tape, C., Liu, Q., Maggi, A., and Tromp, J.: Seismic tomography of the southern California crust based upon spectral-element and adjoint methods., Geophysical Journal International, 180, 433-462, 2010.

Vallée, M. and Douet, V.: A new database of Source Time Functions (STFs) extracted from the SCARDEC method, Physics of the Earth and Planetary Interiors, 257, 149-157, 2016.

Virieux, J.: SH-wave propagation in heterogeneous media: Velocity-stress finite-difference method, Geophysics, 49, $1933-1942,1984$.

Yomogida, K.: Fresnel zone inversion for lateral heterogeneities in the Earth, Pure and Applied Geophysics, 138, 391-406, 1992.

Yoshizawa, K. and Kennett, B. L. N.: Multi-mode surface wave tomography for the Australian region using a 3-stage approach incorporating finite-frequency effects, Journal of Geophysical Research, 109, doi:10.1029/2002JB002 254, 2004.

Zhu, H., Bozdağ, E., Peter, D., and Tromp, J.: Structure of the European upper mantle revealed by adjoint tomography, Nat. Geosc., 5, 493-498, 2012. 